\title{
Low Cross-Polarization Improved-Gain Rectangular Patch Antenna
}

\author{
Anurag Singh ${ }^{1, *,+}$ (D) Sandip Vijay ${ }^{2,+}$ and Rudra Narayan Baral ${ }^{1,+}$ \\ 1 Electronics and Communication Engineering Department, Dr. APJ Abdul Kalam Technical University, \\ Lucknow, Uttar Pradesh-226031, India; r.n.baral@gmail.com \\ 2 Electronics and Communication Engineering Department, Institute of Chartered Financial Analysts of \\ India University, Dehradun, Uttarakhand-248197, India; vijaysandip@gmail.com \\ * Correspondence: agill56@gmail.com \\ + These authors contributed equally to this work.
}

Received: 2 September 2019; Accepted: 15 October 2019; Published: 18 October 2019

\begin{abstract}
In this paper, a low cross-polarization improved-gain rectangular patch antenna is presented. A patch-ground shorting pin with defected patch structure (DPS) is introduced to suppress the cross-polarization level. A High Reflective Frequency Selective Surface (HRFSS) superstrate is designed and placed over the proposed antenna at an optimized position to intensify the gain. To characterize the unit-cell of the superstrate, its transmission characteristics are extracted and discussed. Integration of the superstrate achieves a beam contraction resulting in a gain enhancement to $10.65 \mathrm{dBi}$. The proposed antenna has perfect broadside radiation with a cross-polarization level of below $-30 \mathrm{~dB}$ in the entire half power beamwidth. The prototype of the antenna exhibits good agreement between experimental and simulated results.
\end{abstract}

Keywords: antenna; cross-polarization; defected-patch-structure; frequency selective surface; high-gain; superstrate

\section{Introduction}

The microstrip antenna has many excellent features satisfying the requirements of the current wireless communication system being low-profile, easily manufactured, easy to integrate, with multi-polarization, multi-band operations, etc. However, it has some critical limitations too, such as narrow bandwidth, poor gain and higher cross-polarization [1]. In particular, cross-polarization leading to cross-talk is a severe issue in a communication system.

There is extensive literature on the bandwidth and the gain enhancement techniques. Hence, current generation microstrip antennas are capable of handling ultra-wide bandwidth [2] and exhibiting a peak realized gain above $20 \mathrm{dBi}$ [3]. Among the different techniques of gain enhancement, the metamaterial-based technique is commonly used in the literature to intensify the gain of the antenna. A variety of metamaterial structures are reported in the literature to either intensify or shield the electromagnetic radiation. Double negative metamaterials are useful for backward wave propagation. A single negative metamaterial acts as a perfect reflector and is thus, effective for electromagnetic shielding. However, in many cases the single negative metamaterials are deployed beneath the antenna to reflect radiation back towards the broadside direction to enhance the antenna gain. In [4-6], phase-gradient based multilayered structures are utilized to intensify the radiation of the antenna in a particular direction, leading to excellent directive gain. In [7], Fabry Perot based metamaterials are placed over a conventional rectangular patch antenna and contraction of the main lobe is demonstrated, resulting in an improved gain. Xu et al. have detailed a zero refractive index based metamaterial and demonstrated its lensing ability by contracting the HPBW by $4.9^{\circ}$, and $6.7^{\circ}$ 
in the E and H-planes respectively [8]. They have implemented fractal, spiral, and meandered-based strategies to achieve the 3D anisotropic zero-refractive-index metamaterials. In [9,10], backward-wave propagation is demonstrated using left-handed materials. The metamaterial-based technique is based on, either the zero-refractive index, phase compensation using the gradient refractive index, or the Fabry-perot resonant cavity, where metamaterials are placed at a certain distance from the antenna to direct the radiation in a particular direction. Different metamaterials [4-10] reported in the literature have unique qualities in terms of their structure, design simplicity, and effectiveness. However, there is still scope to develop a simplified metamaterial structure to allow easy fabrication, and integration.

Another critical issue of microstrip antennas is their high cross-polarization level for which very few dedicated techniques are reported in the literature. A higher cross-polarization level results in strong cross-talk [11] and is thus extremely threatening to applications like wireless medical telemetry services (WMTS), medical implant services (MICS), military and avionic communications.

Defected ground structure (DGS) is widely used to suppress the cross-polarization [12-15]. Recently, in [12], Kumar et al. presented a rectangular DGS to suppress the cross-polarization of a graphene-based antenna in the elevation plane. In [13], a dumbbell-shaped DGS is reported to achieve a co-cross-polarization isolation of more than $30 \mathrm{~dB}$ for a rectangular patch antenna. Similarly, in [14,15], different DGS structures are employed to suppress the cross-polarization of rectangular patch antennas. However, a few other techniques such as aperture coupling [16], metamaterial based [17,18], and differential feeding [19], are also reported in the literature to suppress the cross-polarization of non-conventional antennas.

In this work, efforts are made to improve the antenna co and cross-polarization isolation as well as gain of a microstrip antenna. Two independent techniques-a shorted defected patch structure (DPS) and installation of the High Reflective Frequency Selective Surface (HRFSS) superstrate are reported to improve the cross-polarization and the gain of the antenna respectively. However, the idea of slot-loaded patch is well-known to obtain the multiband [20], reconfigurable [21], and wideband [22] it is rarely used to suppress the cross-polarization level. In addition, the size of the aperture type unit cell used to develop the proposed superstrate is nearly $27 \%$ compact than the reference [23] unit cell which significantly reduces the overall structure. The proposed work uses the DPS for the narrowband application but it is also effective for the wideband cross-polarization reduction without affecting the gain of the antenna, as reported in [24]. The width of a rectangular patch antenna is shorted to the ground using a shorting-pin and an optimized thin slot is incorporated near to the shorting-pin, resulting in co-cross-polarization isolation of more than $30 \mathrm{~dB}$ in the elevation plane. Moreover, the proposed technique does not disturb the principal pattern of the rectangular patch antenna. Due to the non-conventional patch structure, the feeding location is optimized to perfectly match the $50 \Omega$ SMA connector. Thereafter, an aperture type miniaturized superstrate is installed over the antenna to enhance the gain which is inspired by $[23,25,26]$. As the air-gap affects reflection coefficient [25] of both antenna and the superstrate, optimization of air-gap is required to synchronize the resonant frequency. The height of the superstrate is optimized at $34 \mathrm{~mm}$ achieving best possible synchronization resulting in an excellent peak measured realized gain of $10.65 \mathrm{dBi}$. The characterization of the superstrate is done by analyzing the HRFSS unit cell in the context of transmission characteristics. Finally, a prototype of the proposed technique is developed which is intended to work in the S-band and good agreement between the simulation and measured results have been found.

\section{Defected Patch Antenna}

Ideally, in a conventional rectangular patch antenna, the current covers a half-wavelength path along the length (L) (Figure 1) and radiates only at the edges of the width (W). In a practical situation, some radiation occurs along the length of the patch, caused by the immediate higher-order orthogonal $\mathrm{TM}_{02}$ mode. 


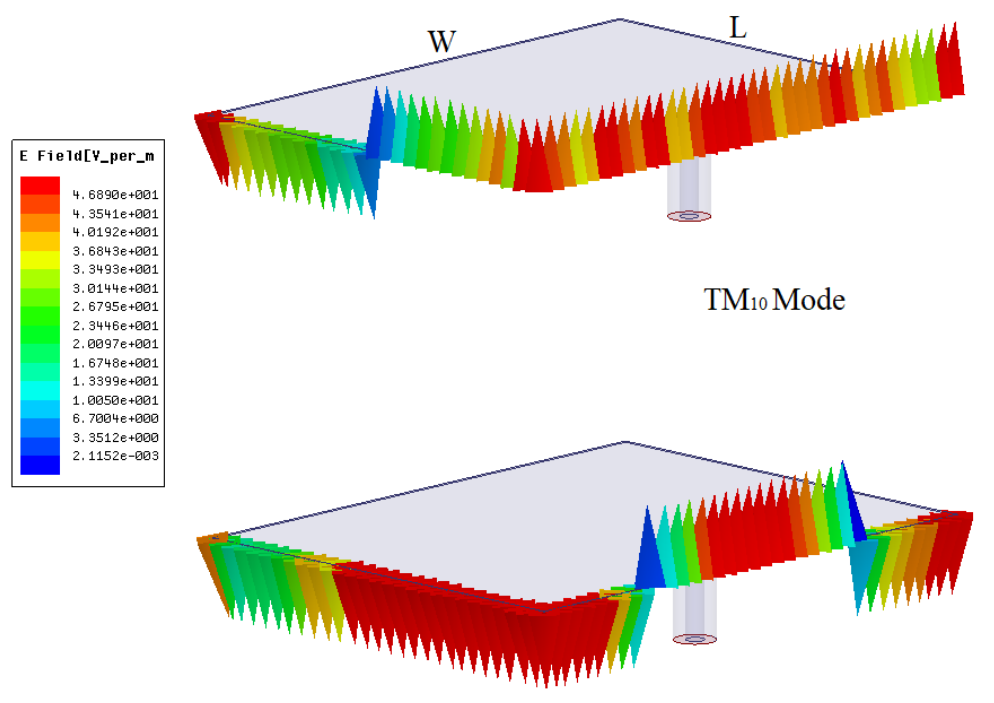

TM02 Mode
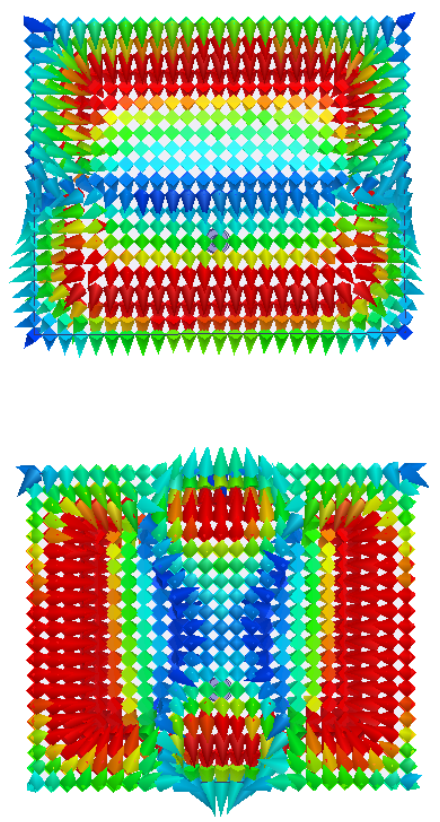

Figure 1. Electric field vectors in dominant $\mathrm{TM}_{10}$ and orthogonal $\mathrm{TM}_{02}$ modes $(2.1 \mathrm{GHz})$.

The electric field variation along the length and width, and into the substrate is shown in Figure 1. However, in the $\mathrm{TM}_{02}$ mode, the current travels along the width and radiates along the length, as shown in Figure 1. It is evident in Figure 1 that in orthogonal $\mathrm{TM}_{02}$ mode, the strongest electric field is present at corners and at a position of $W / 2$. If the position $W / 2$ is shorted to the ground, the radiation caused by the $\mathrm{TM}_{02}$ mode can be significantly suppressed, resulting in lower cross-polarization for the dominant $\mathrm{TM}_{10}$ mode. Shorting the patch and ground will alter the edge-impedance of the patch, thus, the feeding location should be relocated. In this section, the concept is deployed on a rectangular patch antenna designed using a low-cost FR4 substrate of thickness $1.6 \mathrm{~mm}$, and dielectric loss tangent $(\delta)$ of 0.02 at the $2.2 \mathrm{GHz}$ band. Thereafter, a thin rectangular slot is placed near to the shorting-pin to divert the current along the width, which further enhance the co-cross-polarization isolation. The layout of the antenna is shown in Figure 2. In the first iteration, a conventional rectangular patch antenna is designed using a resonant cavity model [1]. Later, the patch and ground are shorted and the feeding location is optimized to obtain the best possible impedance matching.

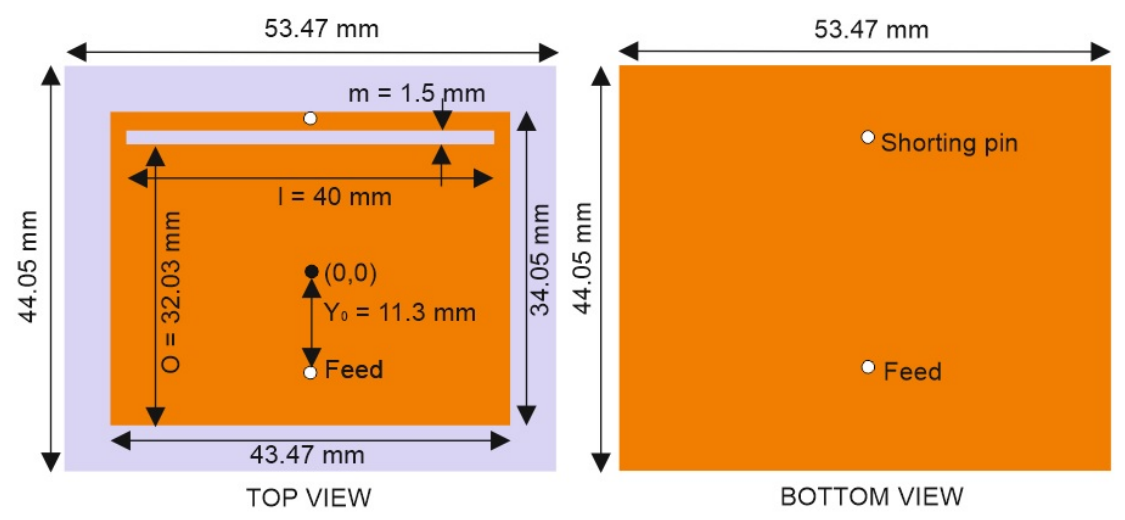

Figure 2. Configuration of the proposed antenna.

The proposed design is evolved in two stages, shorting the patch and ground and incorporating the DPS. The impedance, reflection, gain characteristics of a conventional and the proposed antenna 
with optimized feeding location is shown in Figure 3. It is evident in Figure 3 that, the conventional antenna and the proposed design achieves the best possible impedance matching at 2.1 and $2.2 \mathrm{GHz}$ with different feeding location respectively. However, after implementing the shorting pin and DPS the gain of the antenna is significantly reduced. Initially, a random slot is incorporated into the patch near the shorting pin and the cross-polarization of the rectangular patch antenna is analyzed against the position of the slot $(\mathrm{O})$. The co and cross-polarization levels for the different elevation angles of the proposed antenna against the position of the slot $(\mathrm{O})$ is shown in Figure 4a. It is found that, as the slot shifts closer to the shorting pin, the cross-polarization level improves significantly. However, beyond $\mathrm{O}=32.03 \mathrm{~mm}$, the cross-polarization level starts to degrade, thus, the position of the slot is set to this location. The co and cross-polarization levels have been studied for the elevation angles $\phi-0^{\circ}$, $\phi-90^{\circ}$, and $\phi-135^{\circ}$ and found satisfactory, as shown in Figure 4a. The length of the slot versus the S11 parameter and antenna gain is shown in Figure $4 \mathrm{~b}$.

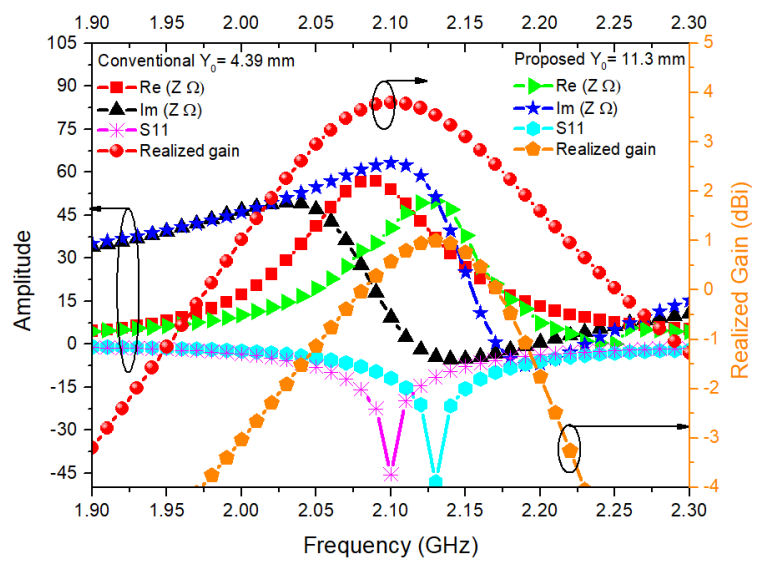

Figure 3. Impedance, reflection, and gain of the conventional and the proposed antenna.

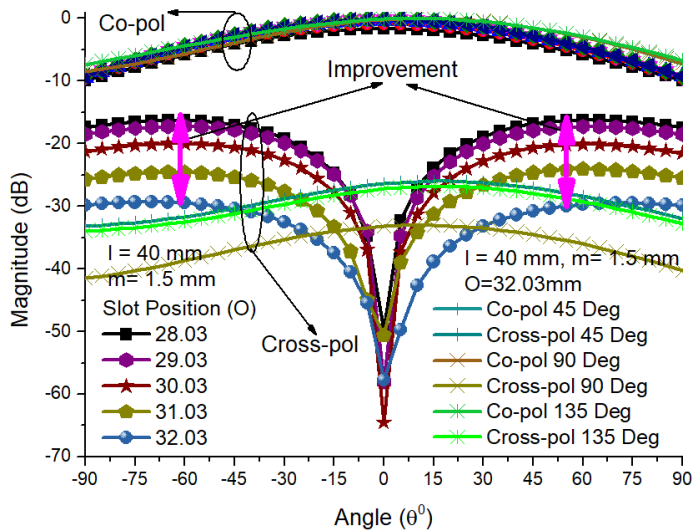

(a)

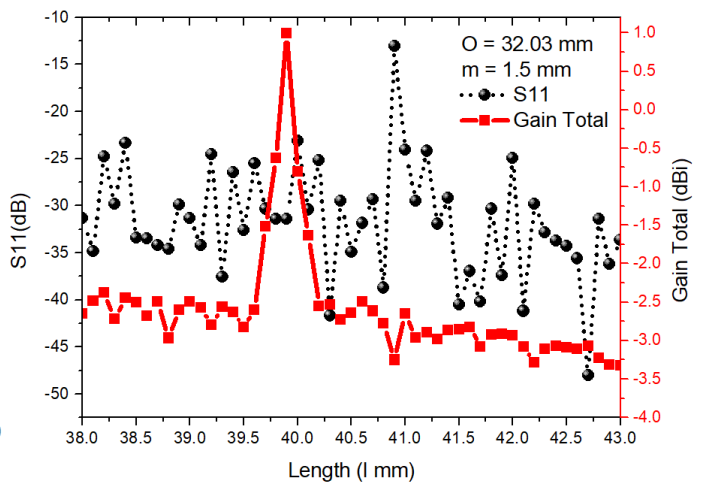

(b)

Figure 4. Parametric analysis at $2.2 \mathrm{GHz}$ (a) Position of the slot $(\mathrm{O})$ versus co and cross-polarization, $\phi=0^{\circ}$ (b) Slot length (l) versus S11 and gain total.

It is clear that the entire range of the slot length (1) satisfies the S11 parameter below $-10 \mathrm{~dB}$, although the gain of the antenna is extremely sensitive to the length of the slot. Thus, very precise optimization is required to achieve the best possible antenna gain. Similarly, using high-frequency-structural-simulator (HFSS) optimetrics, considering the cross-polarization, antenna gain and reflection coefficient the width $(\mathrm{m})$ of the slot is optimized to $1.5 \mathrm{~mm}$. The co-and cross-polarization of the conventional and of the shorted and the final design in different elevation angles and the parametric analysis of slot width $(\mathrm{m})$ and via radius are shown in Figure 5. The co-and 
cross-polarization of the conventional and of the shorted and the final design in different elevation angles are shown in Figure 5a. It is evident in Figure 5 that the shorted ground with the DPS has excellent isolation nearly below $30 \mathrm{~dB}$ in the broaside $\left(\phi-0^{\circ}\right)$. However, for the other elevation angles $\left(\phi-0^{\circ}, \phi-90^{\circ}\right.$, and $\left.\phi-135^{\circ}\right)$ the shorted and shorted with DPS structure have a cross-polarization level difference of around $5 \mathrm{~dB}$. A precise optimization is done against the slot width and via radius which is depicted in Figure 5b,c. It is found that the slot width and via radius is also affecting the CP-XP isolation. An optimum result is obtained for the slot width of $1.5 \mathrm{~mm}$ and via radius of $0.695 \mathrm{~mm}$. While analysing the slot width effect, the distance between the shorting pin and DPS is also considered.

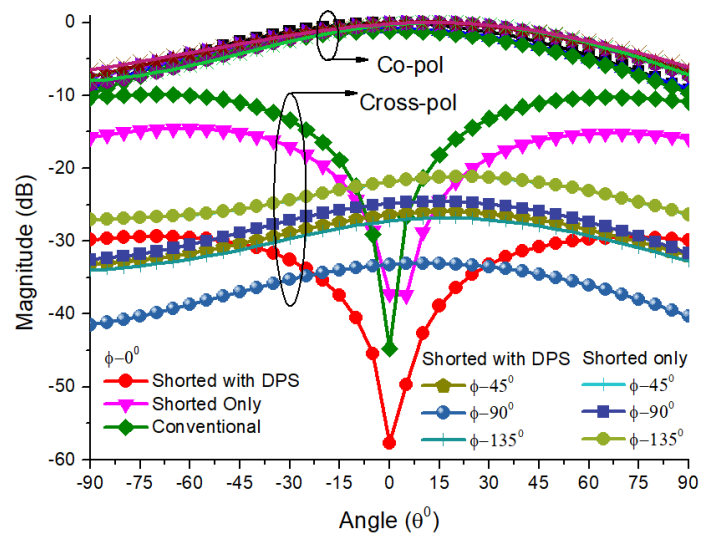

(a)

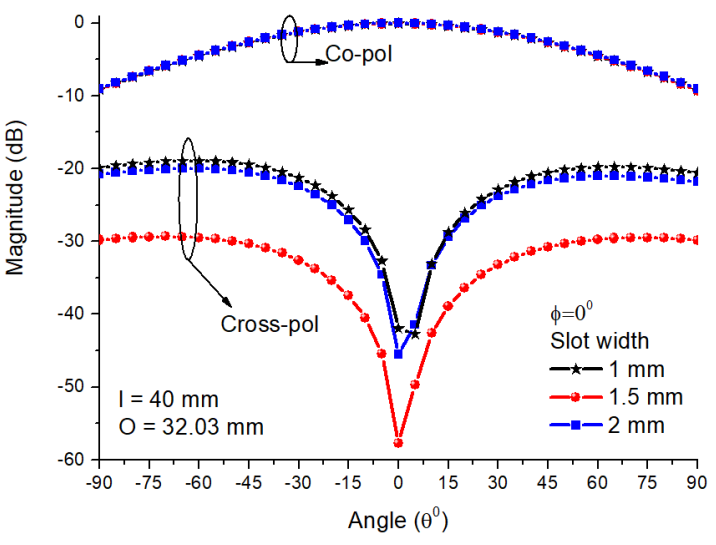

(b)

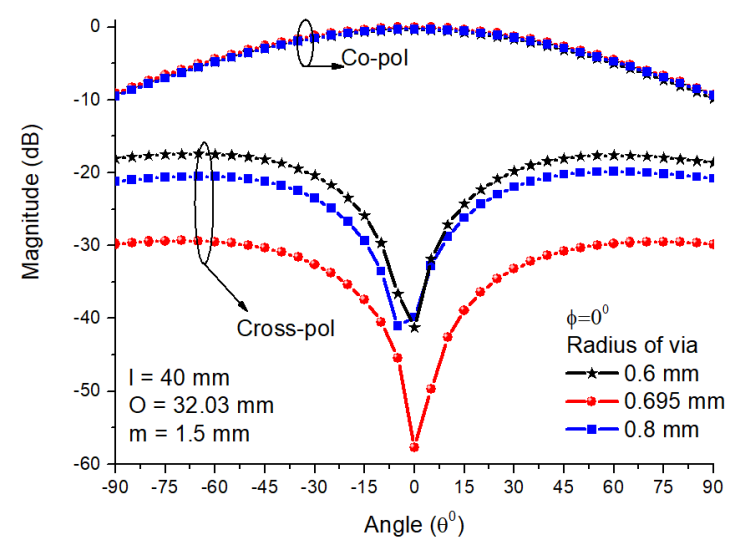

(c)

Figure 5. Parametric analysis (a) Co-cross-polarization isolation of all three iterations, conventional at $2.1 \mathrm{GHz}$, shorted and shorted with defected patch structure (DPS) at $2.2 \mathrm{GHz}$ (impedance matching was disturbed due to the shorting in the second iteration); (b) Effect of slot-width on the cross-polarization; (c) Effect of via-radius on the cross-polarization.

The electric field vectors into the substrate and the surface current distribution of the proposed design are shown in Figure 6. The suppression of the cross-polarization level can be explained by the electric field vectors into the substrate and the surface current distribution, as shown in Figure 6a. In a conventional probe-fed rectangular patch antenna, the cross-polarization components occur at the feeding location as well as at the corners of the patch. It is apparent in Figure 6a that, after implementing the proposed techniques on a conventional rectangular patch antenna, the cross-polarization components at the feeding location and at the corners of the patch are suppressed, resulting in an extremely poor cross-polarization level. Similarly, the surface current distribution shown in Figure $6 \mathrm{~b}$ clearly depicts that the shorting pin attracts maximum current toward it reducing the current flow at corners resulting in improved cross-polarization. 


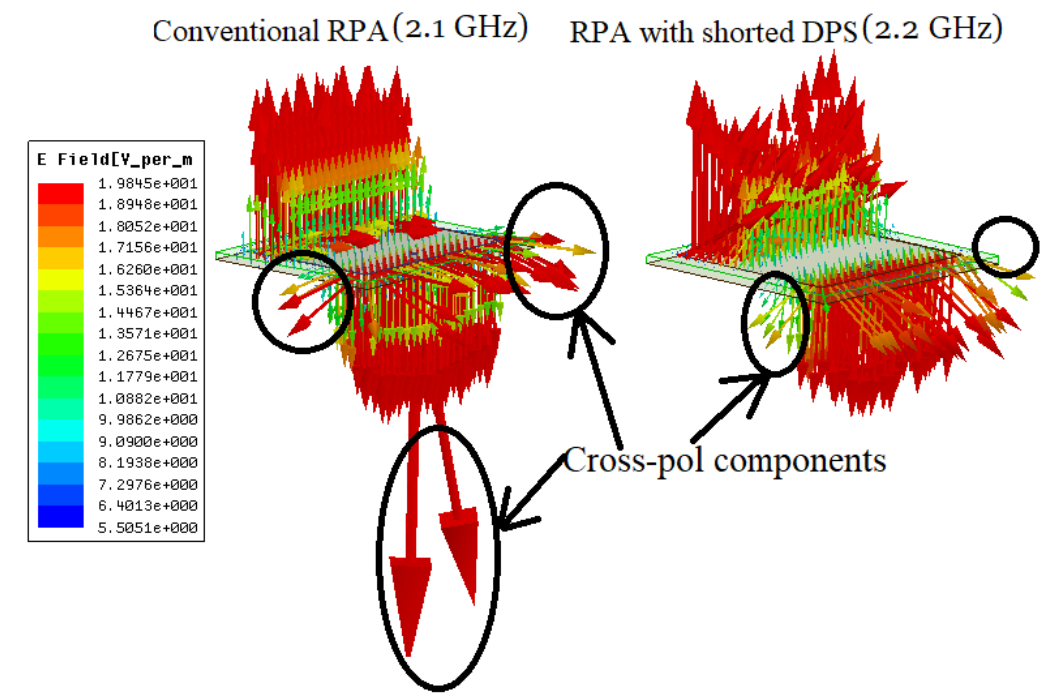

(a)

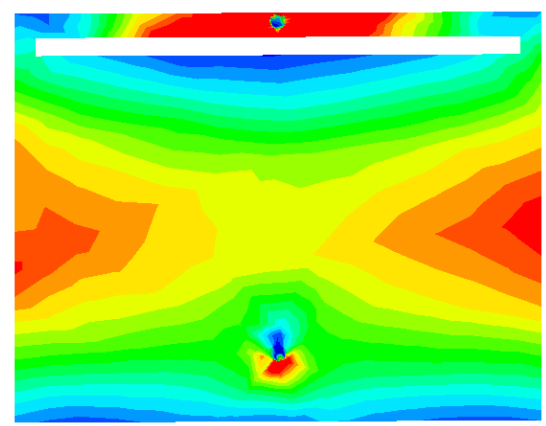

(b)

Figure 6. Cross-polarization analysis (a) Dominant mode electric field vectors of a conventional and the proposed design (b) Surface current distribution on the proposed antenna.

To analyse the effect of the shorting pin, and DPS structure, some basic parameters of the conventional antenna is compared with the proposed antenna, as presented in Table 1 . It can be seen that, the antenna center frequency, and the bandwidth is slightly affected whereas the antenna gain is severely reduced. However, the proposed design achieves an excellent CP-XP isolation of $30 \mathrm{~dB}$. In the following section, a superstrate integrated technique is implemented to further improve the antenna gain.

Table 1. Comparison of the conventional, and proposed antenna structure.

\begin{tabular}{lcccc}
\hline Structure & $\begin{array}{c}\text { Center Frequency } \\
\text { (GHz) }\end{array}$ & $\begin{array}{c}\text {-10 dB Impedance } \\
\text { Bandwidth (MHz) }\end{array}$ & Gain (dBi) & $\begin{array}{c}\text { CP-XP } \\
\text { Isolation (dB) }\end{array}$ \\
\hline Conventional & 2.1 & 60 & 3.8 & 14 \\
Proposed & 2.2 & 64 & 1 & 30 \\
\hline
\end{tabular}

\section{Superstrate Integrated Antenna}

The rectangular patch antenna with DPS has excellent co-and cross-polarization isolation. However, the shorting pin attracts the maximum current towards it which reduces current flow near edges of the width. Thus, a small amount of desired radiations along width is also being eliminated causing a degraded gain of around $1 \mathrm{dBi}$. Hence, to enhance the gain of the antenna, an HRFSS-based superstrate built on $1.6 \mathrm{~mm}$ thick FR4 substrate is installed above the antenna, leading to contraction 
of the main lobe and improved gain. In this section, the superstrate is characterized using transmission parameters and the performance of the superstrate-integrated antenna is discussed. The layout of the unit cell and superstrate is shown in Figure 7.

To analyze the unit cell, periodic boundaries are applied in the HFSS and the scattering parameters are extracted. Later, using the following expressions the transmission $(\mathrm{T})$ and reflection $(\tau)$ coefficients and material parameters are calculated [27]:

$$
\begin{aligned}
& P=\frac{S_{11}^{2}+S_{22}^{2}-\tau}{2 S_{11}^{2}} \\
& \tau=P \pm \sqrt{P-1} \\
& T=\frac{S_{11}+S_{22}-\tau}{1-\left(S_{11}+S_{22}\right) \tau}
\end{aligned}
$$

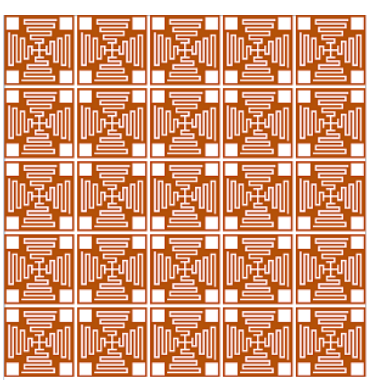

$5 \times 5$ Array

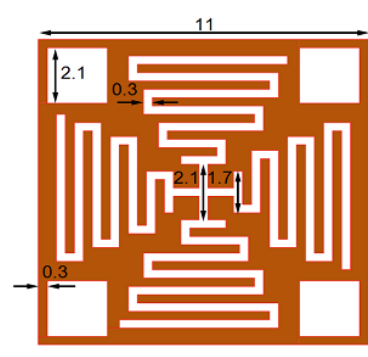

Unit cell

Figure 7. Layout of the superstrate and unit cell (Units mm).

It is evident in Figure 8 that the proposed unit cell supports wideband from $2.1 \mathrm{GHz}$ to $2.54 \mathrm{GHz}$ with excellent transmission coefficient and reflection coefficient below $-18 \mathrm{~dB}$ which thus ensures the Anomalous Refraction (AR) [4]. The reflection phase of the unit cell is also presented in Figure 8 which clearly depicts the three zero-crossing points and also confirms the Anomalous Refraction. The superiority of the proposed superstrate is presented in Table 2. It is clear that the proposed aperture type superstrate is compact and exhibits improved gain compared to the [23].

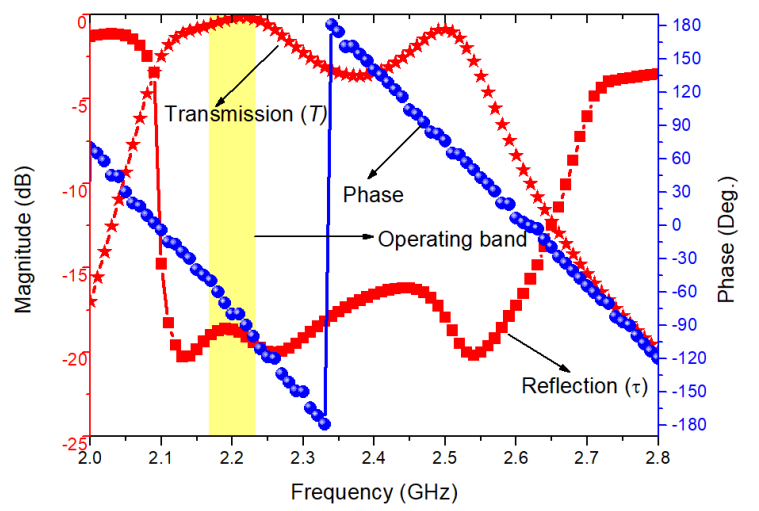

Figure 8. Reflection, transmission, and phase characteristics of the unit cell.

Table 2. Comparison of the proposed superstrate with existing superstrates.

\begin{tabular}{lccccc}
\hline Reference & $\begin{array}{c}\text { Size of the } \\
\text { Superstrate }\end{array}$ & Type of Unit Cell & $\begin{array}{c}\text { Realized } \\
\text { Gain } \mathbf{( d B i})\end{array}$ & $\begin{array}{c}\text { Substrate } \\
\text { Thickness }(\mathbf{m m})\end{array}$ & $\begin{array}{c}\text { Dielectric } \\
\text { Constant }(\varepsilon)\end{array}$ \\
\hline$[23]$ & $1.58 \lambda_{0}$ & Aperture & 8.95 & 1.6 & 4.4 \\
Proposed & $0.43 \lambda_{0}$ & Aperture & 10.65 & 1.6 & 4.4 \\
\hline
\end{tabular}


The proposed antenna is matched to a narrowband from $2.16 \mathrm{GHz}$ to $2.23 \mathrm{GHz}$ which lies within the range of the unit-cell. It is clearly discussed in [23] that a larger superstrate array placed at an optimized height exhibits higher gain/directivity. In this work, to limit the overall size of the structure a small $5 \times 5$ unit cell array $\left(58.6 \times 58.6 \mathrm{~mm}^{2}\right)$ is utilized exhibiting sufficiently measured higher gain of $10.65 \mathrm{dBi}$. In addition, the position of the superstrate significantly affect the resonant frequency and reflection coefficient thus, a precise optimization is required. The optimum distance between the antenna and the structure is estimated to $34 \mathrm{~mm}\left(0.233 \lambda_{0}\right)$ through optimization, as shown in Figure 9. The 3D polar gain total of the conventional rectangular patch antenna, the rectangular patch antenna with the shorted DPS, and the superstrate integrated final design are shown in Figure 10. The contraction of the HPBW and the enhancement of the antenna gain of the superstrate-integrated final design can be clearly observed in Figure 10. The conventional rectangular patch antenna and the shorted rectangular patch antenna with the DPS have nearly equal gain and HPBW. However, the rectangular patch antenna with the shorted DPS has excellent co and cross-polarization, as discussed in the earlier section. The HPBW of the final design is reduced by nearly $67 \%$, so, a simulated total gain of $11.67 \mathrm{dBi}$ is achieved, as shown in Figure 10. To validate the above concept, the prototype of the antenna structure is fabricated and the measured results are compared with the simulated results in the following section.

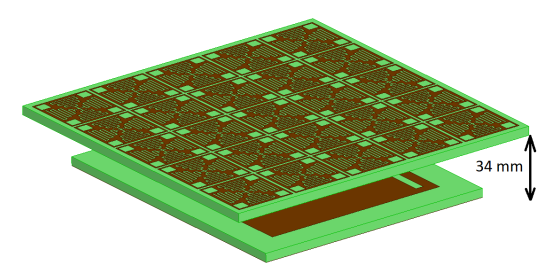

Figure 9. Arrangement of the antenna and the superstrate.

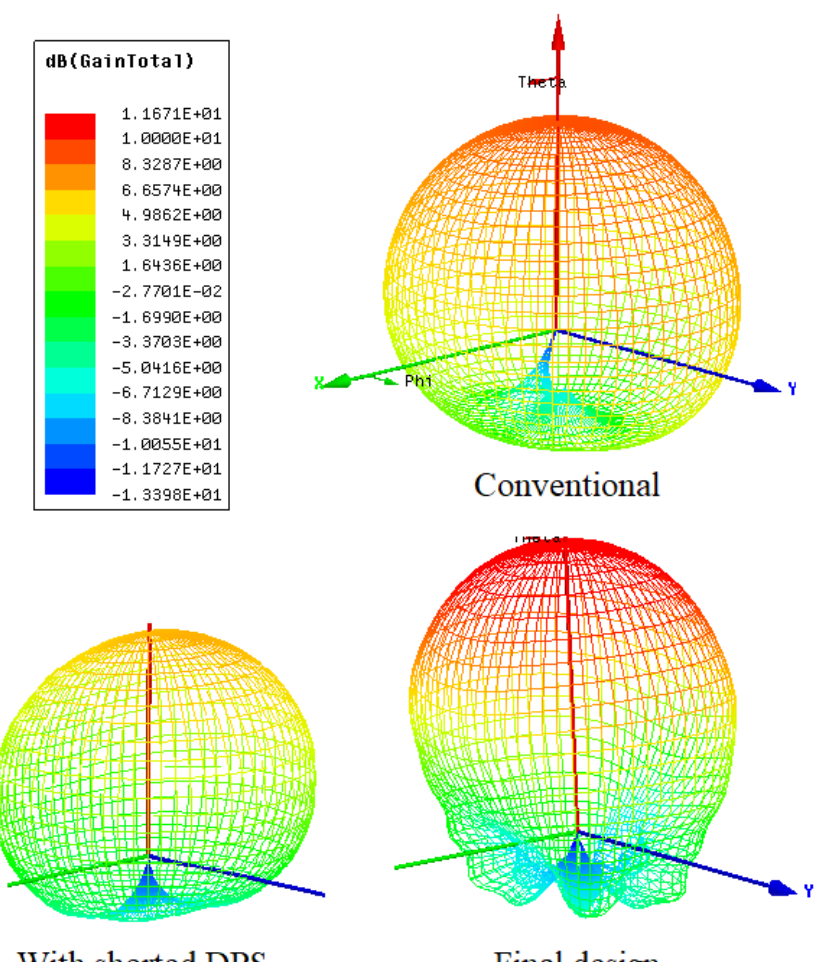

With shorted DPS

Final design

Figure 10. 3D radiation pattern of different configurations. 
The size and the position of the antenna is analysed and shown in Figure 11. It is evident in the Figure 11a that as the size of the superstrate is increasing the gain of the antenna is increasing. Also, for the different superstrate size the center frequency of the overall design is also shifting. Thus, it is essential to precisely optimize the superstrate size before the implementation of the proposed concepts. The simulated realized gain of the propose design against the position of the superstrate of size $5 \times 5$ is shown in Figure 11b. From Figure 11b it is learned that the proposed structure exhibits maximum realized gain when the superstrate is installed at a height of $0.43 \lambda_{0}$.

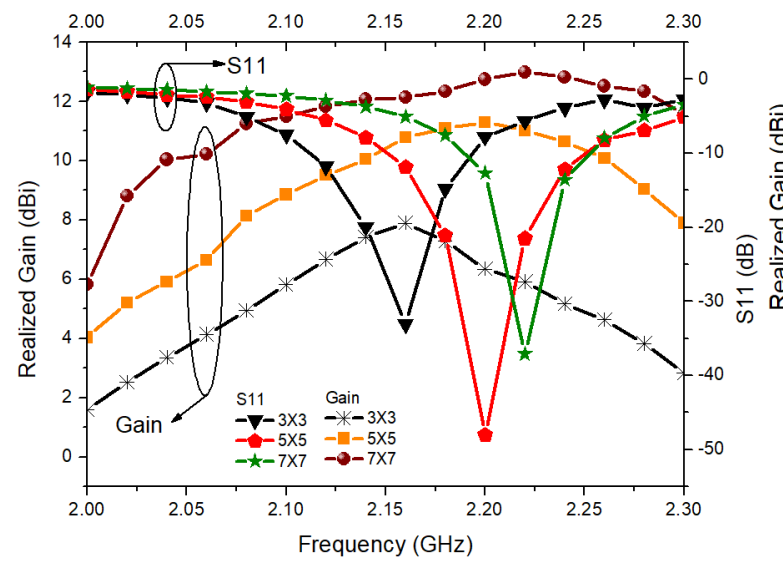

(a)

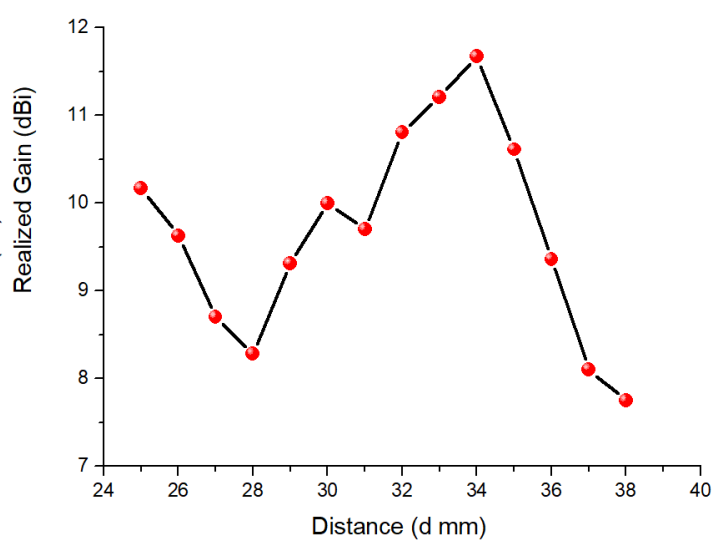

(b)

Figure 11. Optimization of the superstrate integrated design (a) Superstrate size versus gain and S11 (b) Superstrate height versus gain.

\subsection{Prototype and Measured Results}

The prototypes of the superstrate, antenna and superstrate-integrated antenna are shown in Figure 12. The prototype of the proposed design is precisely fabricated, leading to an excellent agreement between the simulation and measured results. The measured results have slight deviations/error ensuring the realization of the proposed design. To install the superstrate over the antenna, four foam pillars of height $34 \mathrm{~mm}$ are pasted at the corners of the antenna, as shown in Figure 12. The S11 parameter and the realized gain of the antenna are measured using a Vector Network Analyzer and the two-antenna method respectively. The radiation pattern of the antenna is measured in an electromagnetically shielded chamber. The simulated and measured S11 parameter of the proposed antenna with and without superstrate are shown in Figure 13. It is clear in Figure 13 that the loading of superstrate is significantly shifting the frequency. A slight shift in the measured S11 parameter of the superstrate integrated configuration can be seen in Figure 13. The main reason for the deviation in the simulated and measured S11 is the mismatch between the dielectric constant in simulation model (ideal considerations, $\varepsilon_{r}=4.4$ ) of the substrate and a practically used substrate. In addition, in the simulation, an ideal $50 \Omega$ port is used to excite the antenna.

In practice, a subminiature version-A (SMA) connector is used to feed the antenna, which includes some losses, leading to a slight mismatch between the simulated and measured results. However, this mismatch can be overcome using high accuracy substrate and feeding connectors. The proposed antenna has a measured $64 \mathrm{MHz},-10 \mathrm{~dB}$ impedance bandwidth covering from $2.169 \mathrm{GHz}$ to $2.233 \mathrm{GHz}$. The broadside co-and cross-polarization radiation patterns of the conventional antenna, the proposed antenna without superstrate, and with superstrate at $2.2 \mathrm{GHz}$ are depicted in Figure 14. It is evident from Figure 14 that there is a considerable agreement between the simulated and measured co-and cross-polarization levels of the superstrate-integrated antenna. 

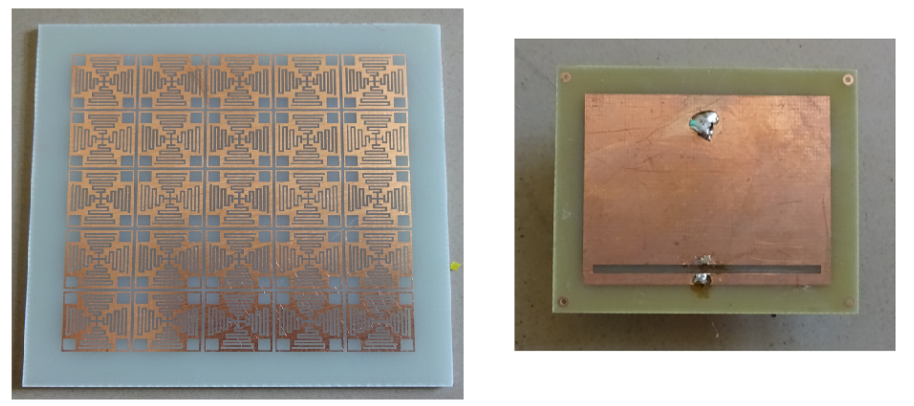

FSS

Antenna TOP

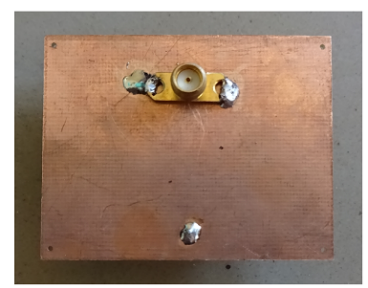

Antenna BOTTOM

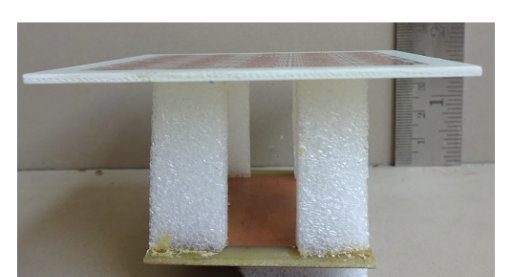

Antenna + FSS

Figure 12. Prototypes of the superstrate integrated antenna.

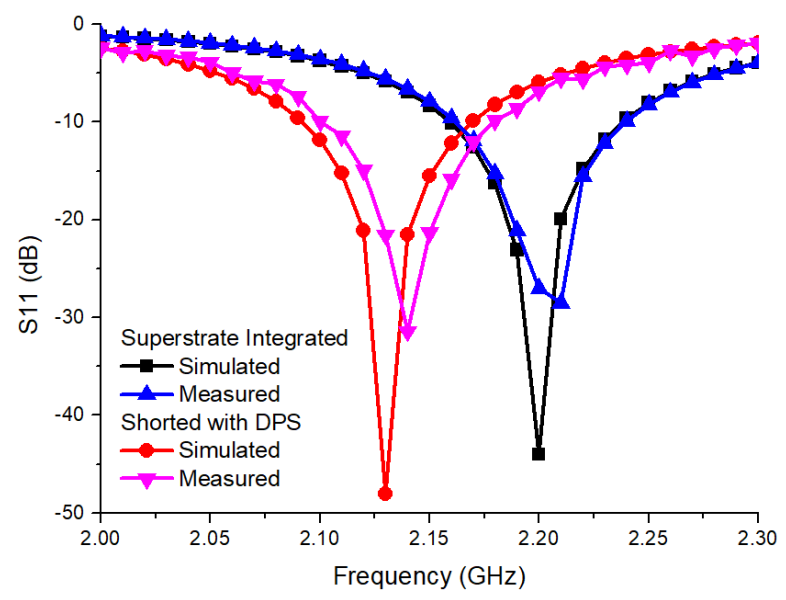

Figure 13. Simulated and measured S11 parameter of antenna element and superstrate integrated antenna.

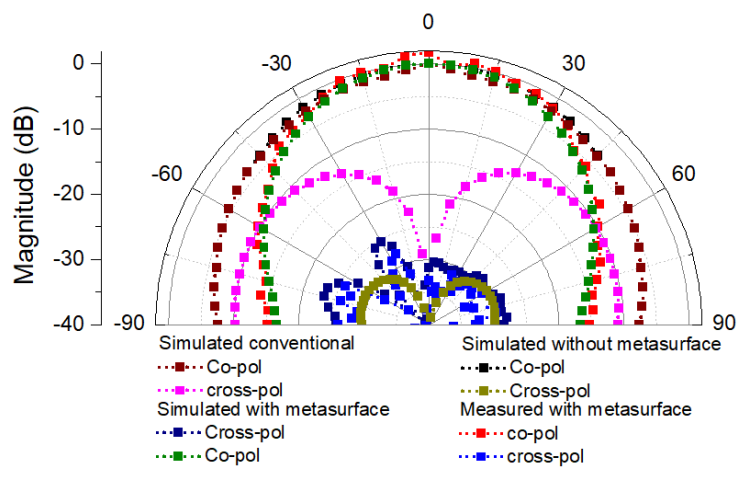

(a)

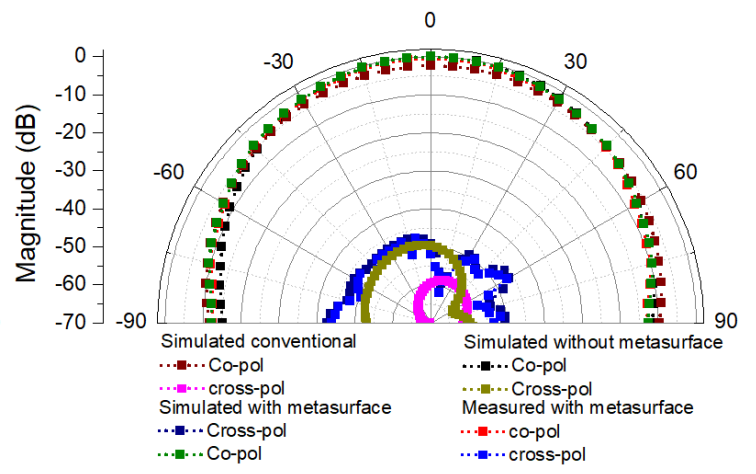

(b)

Figure 14. Broadside radiation pattern of the conventional, with and without metasurface integrated antenna at $2.2 \mathrm{GHz}(\mathbf{a})$ E-plane $\left(\phi-0^{\circ}\right)(\mathbf{b}) \mathrm{H}$-plane $\left(\phi-90^{\circ}\right)$. 
It can be seen in Figure 14a that, after the integration of the slot into the patch and superstrate the cross-polarization level in the H-plane is degraded. However, due to the propagation of the dominant transverse magnetic (TM)-mode, the cross-polarization of the patch antennas is extremely low $(<-40 \mathrm{~dB})$. Hence, this degradation has a negligible effect on the antenna performance.

The measured cross-polarization level in the elevation plane is below $-30 \mathrm{~dB}$, as shown in Figure 14b. However, due to the dominant transverse magnetic mode, the microstrip antennas always have a good co-and cross-polarization isolation level in the azimuth plane. Moreover, for both elevation and azimuth planes, there are considerable fluctuations in the measured cross-polarization levels. This is due to the lower power level of the cross-polarization radiation and the sensitivity of the power meter. The back radiations from the surroundings may also produce an erroneous measured pattern which is more severe at low power level measurement, as cross-polarization. There is a good agreement between simulated and measured co and crosspolarization levels in the E-plane $\left(\phi-0^{\circ}\right)$ and H-plane $\left(\phi-90^{\circ}\right)$. Thus, similar agreement is also expected at $\phi=45^{\circ}$, and $\phi=135^{\circ}$. The E-plane half-power-beamwidth is reduced from $88^{\circ}$ to nearly $40^{\circ}$ after integration of the superstrates which ensures the significant gain enhancement, as depicted in Figure 14a. Whereas, in the H-plane there is a minor improvement. The simulated and measured realized gain of the antenna is shown in Figure 15. It is evident in Figure 15 that the integration of the superstrate is enhancing the gain of the antenna by nearly $9.65 \mathrm{dBi}$ achieving the peak realized gain of $10.65 \mathrm{dBi}$.

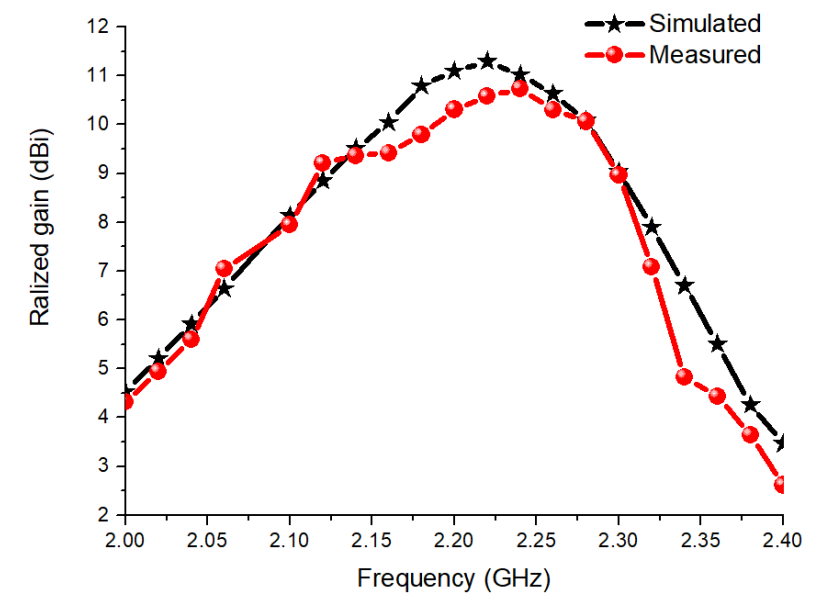

Figure 15. Simulated and measured realized gain.

The proposed structure has excellent co and cross-polarization isolation, improved gain, and narrow HPBW. Thus, it can be used for directive secured communication applications. The proposed work is compared with existing work as reported in Table 3. It is evident in Table 3 that the existing work focuses either on gain enhancement or cross polarization reduction. The high gain antennas $[4,6,8]$ have moderate cross-polarization level of around $-15 \mathrm{~dB}$. Similarly, the low cross polarization antennas [12-15] have moderate gain. However, the antenna proposed in [15] exhibits comparable performance due to the higher operating frequency. In this work, both, gain enhancement and cross-polarization improvement techniques are applied and the remarkable results are obtained. 
Table 3. Comparison of the proposed structure.

\begin{tabular}{lccccccc}
\hline Ref. & $\begin{array}{c}\text { Cross-Pol } \\
\text { Reduction } \\
\text { Technique }\end{array}$ & $\begin{array}{c}\text { Gain Enhancement } \\
\text { Technique }\end{array}$ & $\begin{array}{c}\text { Cross-Pol } \\
\mathbf{( d B})\end{array}$ & $\begin{array}{c}\text { HPBW } \\
\left({ }^{\circ}\right)\end{array}$ & $\begin{array}{c}\text { Gain } \\
(\mathbf{d B i})\end{array}$ & $\mathbf{W} \times \mathbf{L} \times \mathbf{h}\left(\lambda^{3}\right)$ & $\mathbf{F} / \mathbf{B}(\mathbf{d B})$ \\
\hline$[4]$ & Not applied & Phase gradient metasurface & $\approx-15$ & 16.5 & 18.6 & 18.77 & 15.6 \\
\hline$[5]$ & Not applied & $\begin{array}{c}\text { Gradient-refractive-index } \\
\text { metamaterial }\end{array}$ & -13.8 & N/A & 15.26 & 4.45 & N/A \\
\hline$[6]$ & Not applied & $\begin{array}{c}\text { Gradient-refractive-index } \\
\text { metamaterial }\end{array}$ & -15 & N/A & 14 & 2.83 & N/A \\
\hline$[8]$ & Not applied & $\begin{array}{c}\text { Zero-refractive-index } \\
\text { metamaterial }\end{array}$ & -20.8 & 15.6 & 21.25 & 8.6 & N/A \\
\hline$[12]$ & DGS & Not applied & -30 & 88 & N/A & 0.23 & N/A \\
\hline$[13]$ & DGS & Not applied & -30 & $\approx 70$ & 6.13 & 0.38 & N/A \\
\hline$[14]$ & DGS & Not applied & -28 & $\approx 80$ & 2.4 & 0.2 & N/A \\
\hline$[15]$ & DGS & Not applied & -30 & $\approx 70$ & 7.5 & 0.044 & N/A \\
\hline This work & Shorted DPS & HRFSS Superstrate & -30 & 29.92 & 10.65 & 0.046 & 14.4 \\
\hline
\end{tabular}

\section{Conclusions}

A superstrate-integrated low-cross-polarization antenna is presented. Loading the rectangular slot into the patch-ground shorted rectangular patch antenna is an effective way to suppress the cross-polarization level of a rectangular patch-based antenna. The position and dimensions of the slot need to be optimized to obtain the best possible radiation performance and cross-polarization level. To improve the gain of the antenna, an HRFSS is installed over the antenna element, resulting in contraction of the radiation angle; hence, the antenna gain is improved. Finally, the simulated and measured results show good agreement and thus validate the proposed techniques.

Author Contributions: Conceptualization is done by A.S. Data curation is done by S.V. Formal analysis is done by A.S., S.V. and R.N.B. Investigation of the proposed work is done by A.S. under the supervision of R.N.B. The original draft is written by A.S. and the revisions is done and editing is done by S.V. and R.N.B.

Funding: This research received no external funding.

Conflicts of Interest: The authors declare no conflict of interest.

\section{References}

1. Balanis, C.A. Antenna Theory Analysis and Design, 3rd ed.; John Wiley \& Sons: New York, NY, USA, 2012.

2. Hari, S.M.; Jitendra, K.D.; Mahendra, M.S. A slot resonators based quintuple band-notched Y-shaped planar monopole ultra-wideband antenna. Int. J. Electron. Commun. (AEU) 2018, 83, 70-78.

3. Kumar, J. Compact MIMO antenna. Microw. Opt. Technol. Lett. 2016, 58, 1294-1298. [CrossRef]

4. Li, H.; Wang, G.; Xu, H.; Cai, T.; Liang, J. X-band phase-gradient metasurface for high-gain lens antenna application. IEEE Trans. Antennas Propag. 2015, 63, 5144-5149. [CrossRef]

5. Xu, H.-X.; Wang, G.-M.; Tao, Z.; Cai, T. An octave-bandwidth half maxwell fish-eye lens antenna using three-dimensional gradient-index fractal metamaterials. IEEE Trans. Antennas Propag. 2014, 62, 4823-4828. [CrossRef]

6. Xu, H.-X.; Wang, G.-M.; Tao, Z.; Cai, T. High-directivity emissions with flexible beam numbers and beam directions using gradient-refractive-index fractal metamaterial. Nat. Sci. Rep. 2014, 4, 5744. [CrossRef] [PubMed]

7. Zahra, M.R.; Pejman, R.; Arash, V. A novel design of Fabry-Perot antenna using metamaterial superstrate for gain and bandwidth enhancement. Int. J. Electron. Commun. (AEU) 2015, 69, 1525-32.

8. Xu, H.-X.; Wang, G.-M.; Tao, Z.; Cai, T. Miniaturization of 3-D anistropic zero-refractive-index metamaterials with application to directive emissions. IEEE Trans. Antennas Propag. 2014, 62, 3141-49. [CrossRef]

9. Xu, H.-X.; Wang, G.-M.; Qi, M.-T.; Xu, Z.-M. A metamaterial antenna with frequency-scanning omnidirectional radiation patterns. Appl. Phys. Lett. 2012, 101, 133501. [CrossRef] 
10. Xu, H.-X.; Wang, G.-M.; Qi, M.Q.; Li, L.; Cui, T.J. Three-dimensional super lens composed of fractal left-handed materials. Adv. Opt. Mater. 2014, 50, 495-502. [CrossRef]

11. Xu, F.; Wang, H.; Jiu, Y.-Q.; Wang, R.; Deng, Y. Impact of cross-polarization isolation on polarimetric target decomposition and target detection. Radio Sci. 2015, 50, 327-338. [CrossRef]

12. Kumar, J.; Basu, B.; Talukdar, F.A.; Nandi, A. Multimode-Inspired low cross-polarization multiband antenna fabricated using graphene-based conductive Ink. IEEE Antennas Wirel. Propag. Lett. 2018, 17, 1861-1865. [CrossRef]

13. Subhradeep, C.; Abhijyoti, G.; Sudipta, C.; Lolit, K.S. Improved cross-polarized radiation and wide impedance bandwidth from rectangular microstrip antenna with dumbbell-shaped defected patch surface. IEEE Antennas Wirel. Propag. Lett. 2016, 15, 84-88.

14. Chandrakanta, K.; Debatosh, G. Asymmetric geometry of defected ground structure for rectangular microstrip: A new approach to reduce its cross-polarized fields. IEEE Trans. Antennas Propag. 2016, 64, 2503-2506.

15. Chandrakanta, K.; Mahammad, I.P.; Debatosh, G. Microstrip patch with nonproximal symmetric defected ground structure (DGS) for improved cross-polarization properties over principal radiation planes. IEEE Trans. Antennas Propag. 2015, 14, 1412-1414.

16. Lai, C.H.; Han, T.-Y.; Chen, T.R. Broadband aperture-coupled microstrip antennas with low cross-polarization and back radiation. Prog. Electromagn. Res. Lett. 2008, 5, 187-197. [CrossRef]

17. Soumen, P.; Akhilesh, M.; Priyadip, R. A low-profile high-gain substrate-integrated waveguide-slot antenna with suppressed cross-polarization using metamaterial. IEEE Antennas Wirel. Propag. Lett. 2017, 16, 1614-1617.

18. Xu, H.-X.; Wang, G.-M.; Qi, M.Q.; Cai, T. Compact fractal left-handed structures for improved cross-polarization radiation pattern. IEEE Trans. Antennas Propag. 2014, 62, 546-554. [CrossRef]

19. Jin, H.; Che, W.; Chin, K.-S.; Shen, G.; Yang, W.; Xue, Q. 60-GHz LTCC differential-fed patch antenna array with high gain by using soft-surface structures. IEEE Trans. Antennas Propag. 2017, 65, 206-216. [CrossRef]

20. Kumar, J.; Basu, B.; Talukdar, F.A.; Nandi, A. Stable-multiband frequency reconfigurable antenna with improved radiation efficiency and increased number of multiband operations. IET Microw. Antennas Propag. 2019, 13, 642-648. [CrossRef]

21. Kumar, J.; Talukdar, F.A.; Basu, B. Frequency reconfigurable E-shaped antenna for medical application. Microw. Opt. Technol. Lett. 2016, 58, 2215-2217. [CrossRef]

22. Wong, K.-L.; Huang, C.-Y. Triple-wideband open-slot antenna for the LTE metal-framed tablet device. IEEE Trans. Antennas Propag. 2015, 63, 5966-5971. [CrossRef]

23. Peddakrishna, S.; Khan, T.; Kanaujia, B. Resonant characteristics of aperture type FSS and its application in directivity improvement of microstrip antenna. J. Electron. Commun. (AEU) 2017, 79, 199-206. [CrossRef]

24. Ghosh, A. Chattopadhyay, S.; Singh, L.K.L.; Basu, B. Wide bandwidth microstrip antenna with defected patch surface for low cross polarization applications. Int. J. RF Microw. Comput. Aided Eng. 2017. [CrossRef]

25. Foroozesh, A.; Shafai, L. Investigation into the effects of the patch-type FSS superstrate on the high-gain cavity resonance antenna design. IEEE Trans. Antennas Propag. 2010, 58, 258-270. [CrossRef]

26. Jiang, H.; Xue, Z.; Zeng, Q.; Li, W.; Ren, W. High-gain low-RCS slot antenna array based on checkerboard surface, IET Microw. Antennas Propag. 2018, 12, 237-240. [CrossRef]

27. Balanis, C.A. Advance Engineering Electromagnetics, 2nd ed.; John Wiley \& Sons: New York, NY, USA, 2012.

(C) 2019 by the authors. Licensee MDPI, Basel, Switzerland. This article is an open access article distributed under the terms and conditions of the Creative Commons Attribution (CC BY) license (http:/ / creativecommons.org/licenses/by/4.0/). 